\title{
Hypertonic saline in patients with primary ciliary dyskinesia: on the road to evidence- based treatment for a rare lung disease
}

\author{
Claudia E. Kuehni ${ }^{1,2}$, Myrofora Goutaki ${ }^{1,2}$ and Helene E. Kobbernagel ${ }^{3}$ \\ Affiliations: ${ }^{1}$ Institute of Social and Preventive Medicine, University of Bern, Switzerland. ${ }^{2}$ Paediatric \\ Respiratory Medicine, Children's University Hospital of Bern, University of Bern, Switzerland. ${ }^{3}$ Danish
} Paediatric Pulmonary Service, Copenhagen University Hospital, Rigshospitalet, Copenhagen, Denmark.

Correspondence: Claudia E. Kuehni, Institute of Social and Preventive Medicine, University of Bern, Finkenhubelweg 11, CH-3012, Bern, Switzerland. E-mail: claudia.kuehnidispm.unibe.ch

@ERSpublications

Participation in clinical studies should be an essential part of routine care for patients with rare lung diseases http://ow.ly/WfAm307Tqsg

Cite this article as: Kuehni CE, Goutaki M, Kobbernagel HE. Hypertonic saline in patients with primary ciliary dyskinesia: on the road to evidence-based treatment for a rare lung disease. Eur Respir J 2017; 49: 1602514 [https://doi.org/10.1183/13993003.02514-2016].

The study of PAfF et al. [1], published in this issue of the European Respiratory Journal on Rare Disease Day 2017, describes the first randomised clinical trial of inhaled hypertonic saline on quality of life and other outcomes in patients with primary ciliary dyskinesia (PCD). Although the results were negative, the study is a major step towards evidence-based treatment of PCD.

\section{PCD: a rare lung disease}

PCD is a rare inherited disease caused by abnormal ciliary structure and/or function [2, 3]. PCD is heterogeneous: mutations in over 30 genes result in a variety of ultrastructural and functional deficits [4], and its clinical presentation varies broadly [5]. PCD mostly manifests with symptoms of the upper and lower airways, but can involve many other organs with ciliated cells. Its diagnostic work-up, summarised in the recent guideline from the European Respiratory Society (ERS) [4], is hampered by the lack of a diagnostic test that unambiguously confirms or excludes PCD. We currently must employ a combination of technically demanding tests that include measurement of nasal nitric oxide, high-speed video microscopy, transmission electron microscopy, and in some cases genotyping and immunofluorescence of ciliary proteins. Prevalence has been estimated at around 1 in $10000[3,6]$, though it is higher in consanguineous communities [7].

An ERS Task Force in 26 countries studied diagnosis and management of PCD in paediatric centres. It found wide variability in prevalence: usually much less than 1 in 10000 [6]. Care is decentralised and many centres follow only 1-5 patients. Treatment varies both between and within countries, and can involve airway clearance therapy, exercise, administration of acute and prophylactic antibiotics, bronchodilators, inhaled corticosteroids, and the use of recombinant human deoxyribonuclease. Most studies had a mixed population of PCD and other respiratory patients, and PCD often was not diagnosed with state-of-the-art methods. The consensus statement of the Task Force concluded that it could not recommend extrapolating from cystic fibrosis (CF) to PCD, since the pathophysiology of these two

Received: Dec 212016 | Accepted: Dec 222016

Information about the authors' funding has been deposited with the Open Funder Registry.

Conflict of interest: None declared.

Copyright OERS 2017 
disorders differs, and it reported a lack of good evidence for the effectiveness of any treatment. The taskforce also called for large, well-designed, randomised controlled trials that clearly describe patients [2].

\section{What this study did}

The study of PAFF et al. [1] is a collaboration between paediatric and adult pulmonologists. In their randomised double-blind crossover trial, 22 patients received twice-daily inhalation of either hypertonic saline (HS, 7\%) or isotonic saline (IS, $0.9 \%$ ), and then after 4 weeks repeated the inhalation procedure with the other solution. Primary outcome was a change in health-related quality of life (the overall score of the St. George's Respiratory Questionnaire, SGRQ). Secondary outcomes were SGRQ subscores, the Quality-of Life Questionnaire-Bronchiectasis (QOL-B), lower respiratory tract symptoms, exacerbations, spirometry, systemic and sputum inflammatory markers, adherence, and adverse events. The carefully designed study, reported according to CONSORT criteria, has many strengths:

1) It is the first clinical trial to include only patients with confirmed PCD.

2) The order of treatment was randomised and allocation was concealed from investigators, patients, and treating physicians throughout the study.

3) The taste of the HS solution was masked (in both solutions) by quinine sulfate.

4) Results were analysed taking intention-to-treat and per-protocol approaches.

5) The target sample size ( 24 patients) was based on the minimal clinically important difference (MCID) of the main outcome (a 4-point reduction in SGRQ total score), and accounted for possible withdrawals.

\section{What the study found}

Patient recruitment was difficult. Of the 86 patients invited to participate, three did not meet the inclusion criteria and 61 declined to participate due to personal reasons, inconvenience, or their desire to not change their current medication.

Results were mostly negative. Although quality of life (SGRQ total score) improved more after HS inhalations ( -2.6 points) than after IS inhalations ( -0.3 points), the difference neither reached the 4 -point MCID nor was statistically significant. Also, results for most secondary endpoints were negative with the exception of the QOL-B Health Perception Scale and the Lower Respiratory Tract Infections-Visual Analogue Scale for chest pain. Given the number of comparisons (41), chance could explain these findings. Adverse events were more common after HS but were mild (throat irritation, cough, chest tightness).

How can we explain the negative findings? Hypertonic saline may not help patients with PCD, at least to a clinically significant extent. But there are alternative explanations.

1) The active and control treatments might not have been sufficiently distinct: isotonic saline could also benefit patients by humidifying airway surface liquid [8], and quinine sulfate, the bitter compound used for taste-masking in both groups, has been reported to stimulate ciliary frequency and have bronchodilatory effects $[9,10]$.

2) HS might not have been administered in the most effective way. Quality of life in PCD is also influenced by ear, nose and throat (ENT) problems [11]. Administration of HS via a face mask instead of a mouthpiece might have been more effective. Combination with intense physiotherapy or an exercise programme to facilitate expectoration of the liquefied sputum might have made inhalations more effective.

3) The outcome measures used in the study were not very sensitive. The SGRQ was designed for patients with asthma and chronic obstructive pulmonary disease; it does not include symptoms typical for PCD such as chest congestion. This might explain why QOL-B, designed for bronchiectasis patients, indicated better outcomes. But even QOL-B excludes ENT symptoms. Future trials will benefit from the new PCD-specific instrument (QOL-PCD), which it is hoped will be more sensitive [12, 13]. Also, spirometry (forced vital capacity, forced expiratory volume in $1 \mathrm{~s}$ and forced expiratory flow at $25-75 \%$ of FVC) was used to measure lung function, but spirometry measures mainly proximal obstruction. Patients with PCD, like those with CF, primarily have peripheral airway obstruction that is picked up much better by gas washout techniques [14-16].

4) Although the study nearly reached the target sample size, it was underpowered mainly because observed variability of the outcomes was larger than anticipated. The detailed data on the variability observed (online table E5 of PAFF et al. [1]) will help the planning of future studies.

Improving treatment for other rare diseases: cystic fibrosis and paediatric cancers The problems encountered in this study hamper evidence-based treatment, but are typical for research on rare diseases. Management of some rare diseases has progressed more than others. 
Among pulmonary disorders, CF stands out. During recent decades we have seen major advances in standardisation of diagnostics and management of CF [17], early diagnosis and treatment thanks to newborn screening [18], centralised treatment in experienced clinics with multidisciplinary teams [19], development of disease-specific outcome measures [20], and randomised controlled trials [21]. Together, these efforts have fantastically improved long-term CF survival to a median age greater than 40 years in developed countries [22]. But why CF, and not also PCD? While CF is rare, it is more common than PCD, affecting 1 in 3000 persons. CF is diagnosed with simple and reliable diagnostic methods, and it has a severe disease course from early life, which leads to increased awareness. In PCD, serious complications such as bronchiectasis develop mostly in adulthood when patients are dispersed among numerous adult specialists who each care for only one or two PCD patients in a lifetime. Finally, patient organisations have played a major role in advancing CF research, and CF patients seem to be more willing to participate in clinical studies (based upon personal experience seeing both PCD and CF patients in a large clinic in Copenhagen).

Perhaps the most successful precedent in clinical study of rare disease is childhood cancer. Although childhood cancers are rare, affected children have been systematically included in international clinical studies since the 1970s [23]. These are usually Phase 3 trials in which the control arm receives state-of-the-art therapy typically including a combination of several chemotherapeutic drugs, radiotherapy, and surgery. One or more experimental arms differ in one or more aspects by including new drugs or varying modes of applications of old drugs, or by stratifying patients into risk groups with different levels of treatment. A study usually includes patients with a specific type of cancer and paediatric oncologists strive to enter every patient into a study. When this is not possible, children are treated according to the control arm of the current study. The best arm in each study is then used as the control arm for a next-generation trial. Recruitment into these trials is high and a large majority of families give consent [24]. Successive studies since the 1960s have led to continuous improvement in treatment; survival rates have increased from $10 \%$ in the 1960 s to over $85 \%$ [23]. Notably, almost all improvement has been obtained by combining common therapies in the most effective manner, rather than by using new drugs. While current studies still aim to optimise treatments, they pay increased attention to reducing acute and long-term treatment side effects. The first paediatric oncology trials were done in national networks, but current studies are usually international [23].

\section{BOX 1 Proposed key elements for improving treatment of patients with rare lung diseases}

\section{International and national management plans}

Every country should have national guidelines that contain specific standards for age-appropriate treatment and care for children and adults with rare lung diseases. These should be based on international evidence-based guidelines, but account for local varying factors.

Disease registration

Every country should support prospective national and international registration and follow-up of children and adults with rare lung diseases.

\section{Access to specialists}

Defined referral pathways should exist so that every patient has access to innovative, age-appropriate treatment managed at a specialised treatment centre. Transition from paediatric to adult care centres should be established.

\section{Multidisciplinary teams}

Every child and adult with a rare lung disease should be treated by a multiprofessional team, which treats a sufficient number of patients with the same disease to maintain the necessary skills and participates in auditing and accreditation schemes.

\section{Standardised treatment}

Every patient diagnosed with a rare lung disease for which an observational or interventional clinical study is available should be entered into the relevant study at time of diagnosis. This will enable the patient to profit from the best available treatment. If formal participation in a study is not possible, patients still should be treated according to that study's protocol whenever possible.

\section{Research}

A national strategy is needed to ensure support for investigator-led clinical and translational research, with the recognition that participation of patients in clinical trials is integral to delivery of best-practice care for people with a rare lung disease.

Family support and role of patient organisations

Parental and other family support is crucial to treatment outcome of persons with rare diseases. Patient organisations, too, are essential partners when developing and implementing healthcare policies.

Adapted from SIOP Europe's proposed seven key elements for a national cancer strategy. 


\section{How to move forward}

The RCT of PAFF et al. [4] is not a frustrating experience. It provides detailed clinical data for PCD patients, and helps us to design future studies.

1) The pathophysiology of PCD is unique. A treatment that works for CF or non-CF bronchiectasis $[8,25]$ might not for PCD. We must conduct studies including only patients with state-of-the-art diagnoses of PCD [4].

2) We must learn more about variability in outcome measures in patients with PCD. This study's data and further observational data collected in international datasets such as the Provalf cohort planned as part of the European Union COST Action BEAT-PCD [26], the international PCD (iPCD) cohort study [27], and the international PCD registry [28] will be important.

3) We need more sensitive outcome measures such as disease-specific quality-of-life instruments (not yet available when this study was conducted) and sensitive lung function measurements $[14,16]$.

4) We should design interventions that account for the multisystem aspect of PCD. A management strategy that benefits upper and lower airways or combines mucus liquefaction and expectoration might be more effective.

5) To improve recruitment we should organise multinational studies and include children (who account for most diagnosed patients); simplify studies by reducing number of visits, time needed, and invasive measurements; embed studies in routine care; and promote an alliance between patients and doctors to advance knowledge and develop better PCD treatments.

An ongoing multicentre study that evaluates the efficacy and safety of azithromycin as maintenance therapy in PCD, as part of the European Union FP7 project BESTCILIA, has many of these attributes, though it does suffer from recruitment difficulties [29].

To truly improve the evidence base for treatment of PCD and other rare lung diseases, we need major rethinking. We propose some key points (Box 1) borrowed and adapted from the standards of care developed by the European Society for Paediatric Oncology (SIOP) together with patients and parents [23, 30]. We believe that adopting these points could lead to a significant improvement in care for patients with rare lung diseases such as PCD.

\section{Acknowledgements}

C.E. Kuehni is a participant in the European Union's Seventh Framework Programme under EG-GA No. 35404 BESTCILIA: Better Experimental Screening and Treatment for Primary Ciliary Dyskinesia. All authors participate in the network of COST Action BEAT-PCD: Better Experimental Approaches to Treat PCD (BM 1407); C.E. Kuehni and M. Goutaki were members of the ERS PCD Taskforce for PCD Diagnostics (ERS TF-2014-04). C.E. Kuehni receives funding from the Swiss National Science Foundation (32003B_162820). The views expressed in this manuscript do not reflect official views of these collaborations. We thank Christopher Ritter (Institute of Social and Preventive Medicine, University of Bern, Switzerland) for proofreading and editorial contributions.

\section{References}

1 Paff T, Daniels J, Weersing E, et al. A randomised controlled proof-of-concept study on the effect of inhaled hypertonic saline on quality of life in primary ciliary dyskinesia. Eur Respir J 2017; 49: 1601770.

2 Barbato A, Frischer T, Kuehni CE, et al. Primary ciliary dyskinesia: a consensus statement on diagnostic and treatment approaches in children. Eur Respir J 2009; 34: 1264-1276.

3 Lucas JS, Walker WT, Kuehni CE, et al. Primary Ciliary Dyskinesia. In: Cordier J-F, ed. Orphan Lung diseases (European Respiratory Monograph). Sheffield, European Respiratory Society, 2011; pp. 201-217.

4 Lucas JS, Barbato A, Collins SA, et al. European Respiratory Society guidelines for the diagnosis of primary ciliary dyskinesia. Eur Respir J 2017; 49: 1601090.

5 Goutaki M, Meier AB, Halbeisen FS, et al. Clinical manifestations in primary ciliary dyskinesia: systematic review and meta-analysis. Eur Respir J 2016; 48: 1081-1095.

6 Kuehni CE, Frischer T, Strippoli MP, et al. Factors influencing age at diagnosis of primary ciliary dyskinesia in European children. Eur Respir J 2010; 36: 1248-1258.

7 O'Callaghan C, Chetcuti P, Moya E. High prevalence of primary ciliary dyskinesia in a British Asian population. Arch Dis Child 2010; 95: 51-52.

8 Nicolson $\mathrm{CH}$, Stirling RG, Borg BM, et al. The long term effect of inhaled hypertonic saline 6\% in non-cystic fibrosis bronchiectasis. Respir Med 2012; 106: 661-667.

9 Shah AS, Ben-Shahar Y, Moninger TO, et al. Motile cilia of human airway epithelia are chemosensory. Science 2009; 325: 1131-1134.

10 Deshpande DA, Wang WC, McIlmoyle EL, et al. Bitter taste receptors on airway smooth muscle bronchodilate by localized calcium signaling and reverse obstruction. Nat Med 2010; 16: 1299-1304.

11 McManus IC, Mitchison HM, Chung EM, et al. Primary ciliary dyskinesia (Siewert's/Kartagener's syndrome): respiratory symptoms and psycho-social impact. BMC Pulm Med 2003; 3: 4.

12 Lucas JS, Behan L, Dunn Galvin A, et al. A quality-of-life measure for adults with primary ciliary dyskinesia: QOL-PCD. Eur Respir J 2015; 46: 375-383.

13 Dell SD, Leigh MW, Lucas JS, et al. Primary ciliary dyskinesia: first health-related quality-of-life measures for pediatric patients. Ann Am Thorac Soc 2016; 13: 1726-1735. 
14 Boon M, Vermeulen FL, Gysemans W, et al. Lung structure-function correlation in patients with primary ciliary dyskinesia. Thorax 2015; 70: 339-345.

15 Green K, Buchvald FF, Marthin JK, et al. Ventilation inhomogeneity in children with primary ciliary dyskinesia. Thorax 2012; 67: 49-53.

16 Nyilas S, Schlegtendal A, Singer F, et al. Alternative inert gas washout outcomes in patients with primary ciliary dyskinesia. Eur Respir J 2017; 49: 1600466.

17 Smyth AR, Bell SC, Bojcin S, et al. European Cystic Fibrosis Society standards of care: best practice guidelines. J Cyst Fibros 2014; 13: Suppl. 1, S23-S42.

18 Castellani C, Southern KW, Brownlee K, et al. European best practice guidelines for cystic fibrosis neonatal screening. J Cyst Fibros 2009; 8: 153-173.

19 Conway S, Balfour-Lynn IM, De Rijcke K, et al. European Cystic Fibrosis Society standards of care: framework for the cystic fibrosis centre. J Cyst Fibros 2014; 13: Suppl. 1, S3-22.

20 Quittner AL, Buu A, Messer MA, et al. Development and validation of The Cystic Fibrosis Questionnaire in the United States: a health-related quality-of-life measure for cystic fibrosis. Chest 2005; 128: 2347-2354.

21 Döring G, Elborn JS, Johannesson M, et al. Clinical trials in cystic fibrosis. J Cyst Fibros 2007; 6: 85-99.

22 Elborn JS. Cystic fibrosis. Lancet 2016; 388: 2519-2531.

23 Pritchard-Jones K, Pieters R, Reaman GH, et al. Sustaining innovation and improvement in the treatment of childhood cancer: lessons from high-income countries. Lancet Oncol 2013; 14: e95-e103.

24 Pritchard-Jones K, Dixon-Woods M, Naafs-Wilstra M, et al. Improving recruitment to clinical trials for cancer in childhood. Lancet Oncol 2008; 9: 392-399.

25 Elkins MR, Robinson M, Rose BR, et al. A controlled trial of long-term inhaled hypertonic saline in patients with cystic fibrosis. N Engl J Med 2006; 354: 229-240.

26 Rubbo B, Behan L, Dehlink E, et al. BEAT-PCD. Proceedings of the COST action BM1407 inaugural conference BEAT-PCD: translational research in primary ciliary dyskinesia - bench, bedside, and population perspectives. BMC Proc 2016; 10: Suppl 9, 66.

27 Goutaki M, Maurer E, Halbeisen F, et al. The International Primary Ciliary Dyskinesia (iPCD) Cohort: methods and first results. Eur Respir J 2017; 49: 1601181.

28 Werner C, Lablans M, Ataian M, et al. An international registry for primary ciliary dyskinesia. Eur Respir J 2016; 47: 849-859.

29 Kobbernagel HE, Buchvald FF, Haarman EG, et al. Study protocol, rationale and recruitment in a European multi-centre randomized controlled trial to determine the efficacy and safety of azithromycin maintenance therapy for 6 months in primary ciliary dyskinesia. BMC Pulm Med 2016; 16: 104.

30 European standards of care for children with cancer. Warsaw, European Society of Paediatric Oncology, 2009. www.siope.eu/wp-content/uploads/2013/09/European_Standards_final_2011.pdf Date last updated: October 14, 2009. 